\title{
PRODUCTION, CHARACTERIZATION, AND MECHANICAL PROPERTIES OF STARCH MODIFIED BY OPHIOSTOMA SPP.
}

\author{
Chun Bei Huang, ${ }^{a}$ Robert Jeng, ${ }^{\mathrm{b}}$ Mohini Sain, ${ }^{\mathrm{b}}$ Bradley A. Saville ${ }^{\mathrm{a}^{*}}$ and Martin Hubbes ${ }^{\mathrm{b}}$
}

\begin{abstract}
Microbial modification of starch with Ophiostoma spp. was investigated, with the purpose of developing a novel packaging material for the food or pharmaceutical industries. Various starch sources, such as tapioca, potato, corn, rice and amylopectin were tested as raw materials. The initial screening demonstrated that tapioca and potato starch had better performance for biopolymer production. The yield was about $85 \%$. Preliminary characterization of the modified biopolymer was also conducted. Following microbial conversion, the percentage of molecules with molecular weight (abbreviated $\mathrm{Mw}$ ) more than 10M (abbreviations of million) Daltons increased from $25 \%$ to $89 \%$ after 3 days, confirming that the modification increased the weight of the starch polymer. Fourier Transform Infrared (FT-IR) revealed changes in the chemical structure of the starch after the modification. Both pure starches and the modified biopolymers were cast into films and tested for mechanical properties. The tensile tests showed that after treatment with the fungus, the peak stress and modulus of the films increased about 10 and 40 times, respectively. Also, the water barrier property was improved. Therefore, microbial modification positively impacted proper-ties relevant to the proposed application. Although the role of the fungus in the modification and the function-property relationship of the biopolymer are not yet completely clear, the results of this study show promise for development of a novel biopolymer that competes with existing packaging materials.
\end{abstract}

Keywords: Ophiostoma spp., Microbial conversion, Polysaccharide, Starch films, Packaging material, GFC, FT-IR

Contact information: a: Department of Chemical Engineering, University of Toronto, Toronto, Ontario, M5S 3E5, Canada; b: Faculty of Forestry, University of Toronto, Toronto, Ontario, M5S 3B3, Canada;

*Corresponding author: : saville@,chem-eng.utoronto.ca

\section{INTRODUCTION}

An increased emphasis on sustainability, eco-efficiency, and green chemistry has driven a search for renewable and environmentally friendly resources. Starch is a biodegradable polysaccharide, produced in abundance at low cost, and exhibits thermoplastic behavior. Therefore, it has become one of the most promising candidates as an alternative material to replace traditional plastics in certain market segments, such as the food packaging industry. Numerous studies have been conducted to optimize the performance of the starch-based plastics (Mali et al. 2004; Soest et al. 1997; Fama et al. 2005; Lawton 1996). Briefly, these studies have shown that important properties for evaluation of a packaging material include mechanical properties, gas and water vapor permeability, thermoforming properties, resistance, transparency, and availability (Weber

Huang et al. (2006). "Starch modified by Ophiostoma spp.," BioResources 1(2), 257-269. 257 
et al. 2001). However, the design and engineering of a starch-based packaging product that possesses all of these required properties is a significant challenge. Difficulties were encountered with cost, and technical hurdles such as brittleness associated with high loads, and poor water and gas barrier properties have to be overcome to finally commercialize the biomaterial (Lorcks 1997). Numerous studies have aimed to modify the functional properties of the starch to enhance the inherent bonding strength. Currently, most of the studies are focused on incorporating additives, such as plasticizers, to improve the performance of the material (Poutanen et al. 1996; Laohakunjit et al. 2003).

It has been reported that certain fungi have the ability to produce exopolysaccharides that have great potential for use in cosmetic and food industries because of their possible bioactive characteristics, rheological behavior, and high stability at high temperature (Selbmann et al. 2003). Ophiostoma spp. was isolated from diseased Elm trees. This fungus is unique, as its natural habitat resides in xylem fluid. Preliminary laboratory studies demonstrated that $O$. spp. can produce exo-polysaccharides in the culture medium (Jeng et al. In press). When starch was used as the substrate in $O$. spp. medium, a biopolymer was produced that showed some characteristics well suited for a bio-packaging application. Therefore, a key objective of this study is to confirm that the fungus is capable of utilizing and modifying starch into a biopolymer with superior mechanical properties compared to starch, while identifying the impact of fermentation conditions.

The scope of the present work is to characterize the biopolymer derived from modification of different starch sources (corn, potato, tapioca and rice) by $O$. spp., and ultimately, develop a commercially viable process for large scale production of a biopolymer that can be used as packaging material for food or even medical applications. In these studies, the resulting biopolymers were characterized by gel-filtration chromatography (GFC) and Fourier Transform Infrared (FT-IR) to determine the changes in the biopolymer following fermentation. Films were also cast to test the mechanical properties of the polymer. Laboratory scale production was carried out in a $10 \mathrm{~L}$ carboy to investigate the compositional changes of the starch polymer during fermentation. Finally, it has not been previously reported that a fungus could be used to modify starch and improve its attributes as a bioplastic. Therefore, this research represents a paradigm shift in the realm of starch-derived plastics, and will provide valuable information regarding the starch modification process and its impact on the final product.

\section{MATERIALS AND METHODS}

\section{Materials}

The Ophiostoma spp. used in this study was supplied by the Great Lakes Forest Center, Canada Forest Service, Sault Ste. Marie, Ontario. Potato, rice, and tapioca starch were all normal commercial grade starch, purchased from the market. Corn starch was obtained by grinding dry kernels of food grade corn. Amylopectin was reagent grade, purchased from Sigma. Glycerol was reagent grade purchased from Fisher.

Huang et al. (2006). "Starch modified by Ophiostoma spp.," BioResources 1(2), 257-269. 258 


\section{Starch Modification}

\section{Biopolymer production}

$25 \mathrm{~g}$ of starch was weighed into a 2-liter flask with $1000 \mathrm{ml}$ of distilled water containing $2 \mathrm{~g}$ of yeast extract and $10 \mathrm{~g}$ of glucose. The medium was then sterilized at $121{ }^{\circ} \mathrm{C}$ for $20 \mathrm{~min} .100 \mathrm{ml}$ of fungal spores (300 to $400 \mathrm{mg}$ in dry weight) was inoculated into the starch suspension. The cultures were maintained as a shake culture $(150 \mathrm{rpm})$ at $25^{\circ} \mathrm{C}$ for 4 days. Polymer was obtained by adding an equal volume of $95 \%$ ethanol to spore-free culture medium. The precipitated polymer was then washed with ethanol, lyophilized, and weighed. Substrates used in this study included starch from corn, potato, tapioca, rice, and amylopectin. The production rate of biopolymer from each feedstock was calculated.

The same procedure, but without the fungal inoculation, was also conducted using tapioca starch as a control. The precipitate, extracted by ethanol, was collected and lyophilized.

\section{Time course of biopolymer production}

Laboratory scale fermentation was also conducted in a 10 liter Nalgene carboy containing 7 liters of starch suspension. To determine the time course of biopolymer production, $1000 \mathrm{ml}$ of spore-free culture filtrate was withdrawn daily from day 1 to day 4. Polymer was recovered by the methods described above, and then characterized.

\section{Characterization of the biopolymer}

\section{Gel-filtration chromatography (GFC)}

GFC was used to determine the molecular weight of the biopolymer and its molecular weight distribution. A TSK-GEL 4000 PWXL column from Tosoh Science was attached to the GFC system, which was equipped with Shimadzu-6A column oven, Perkin Elmer Series 200 pump, Perkin Elmer Advanced LC sample processor ISS200, and Shodex RI-71 refractive index detector. The Totalchrom software was used.

The HPLC operated at a flow rate of $1.0 \mathrm{ml} / \mathrm{min}$ and at ambient temperature. The run time for each sample was 15 minutes. Dextran standards from Sigma with $\mathrm{Mw}$ from 1270 to $24 \mathrm{M}$ were injected to obtain the standard curve.

Approximately $0.5 \mathrm{~g}$ of modified starch sample was put into a small flask and mixed in about $2 \mathrm{ml}$ of water. The flask was shaken using an Orbit shaker, at the speed of $1500 \mathrm{rpm}$ for at least 3 hours. The supernatant was withdrawn and centrifuged before analysis through the GFC column.

\section{Fourier transform infrared (FT-IR)}

Infrared spectra of the samples were recorded on an ATR-FTIR (Bruker Tensor 27) equipped with an MCT detector. The sample pellets were prepared by mixing the fine sample powder with $\mathrm{KBr}$ (Sigma-Aldrich, FT-IR grade). Each spectrum obtained wavenumbers from $400 \mathrm{~cm}-1$ to $4000 \mathrm{~cm}-1$, representing the average of 64 scans. All optical measurements were performed at room temperature under ambient conditions. 


\section{Film Casting}

The generic film formulation was constant for all the films produced, which contained $3.9 \mathrm{~g}$ of the "polymer" sample (biopolymer, control or pure starch) in water. Around $100 \mathrm{ml}$ of water was added and heated for an hour. The solution was then poured into a $15 \mathrm{~cm}$ diameter Petri-dish. The dish was first left at room temperature for 4-5 hours, and then placed into a $35^{\circ} \mathrm{C}$ oven to dry. After $48 \mathrm{hrs}$, the translucent film was removed from the dish for further tests.

\section{Tensile Test}

The films were stored at $35^{\circ} \mathrm{C}$ in the oven for at least two weeks before the tensile test. Five "dog bone" shaped specimens (ASTM D638, type I) were cut from each film. Each specimen had a width of $3.00 \mathrm{~mm}$, and the thickness was measured at a minimum of 5 locations with a caliper gauge. The smallest number was recorded as the thickness of the specimen. Most of the specimens had thicknesses between $0.19 \mathrm{~mm}$ and $0.26 \mathrm{~mm}$.

Tensile tests were completed using a Sintech Universal Tensile Test Machine Model \#1. The gage length was $25.4 \mathrm{~mm}$. The specimen was fixed into the slit and pulled apart by the machine at $2.5 \mathrm{~mm} / \mathrm{min}$, until failure occurred or the preset time was reached, usually 5 minutes. Each test was run in duplicate; the total number of impact tests ranged from two to ten for each type of starch/biopolymer, depending on the availability of the samples. The data for peak stress, elongation at break, and modulus of the films, which represent the strength, elasticity and elasticity in tension of the material, were recorded and analyzed. All of the tests were carried out in a climate controlled room at $23^{\circ} \mathrm{C}$ and $50 \% \mathrm{RH}$.

\section{Water Absorbance Test}

The weight of each of the cast film sample was measured. The films were then soaked in water at ambient temperature. Every 60 minutes, the films were taken out, dried with paper towel, and weighed. The process was repeated until the weight of the films stopped increasing. The water absorbance was calculated from the final weight of the water-laden sample relative to the weight of the original film sample. These data are reported as water absorption per gram of the biopolymer film.

\section{RESULTS AND DISCUSSION}

\section{Starch Modification/Biopolymer Production}

Starch is a complex homo-polymer composed of $\alpha$-D-glucose units that are linked together in two different forms, the linear form amylose and the highly branched amylopectin (Soest et al. 1997). The composition and structure of starch granules vary considerably between different plants, affecting the properties and functions of starches from different crops (Jobling 2004). Therefore, starches from various botanical sources were adopted as testing materials in the microbial modification, including corn kernels, potato starch, wheat flour, tapioca starch, rice starch, and millet starch. Among them, corn, potato, and tapioca were selected for further study after a pre-screening experiment, because they showed superior overall properties compared to the others. By increasing 
the amount of starch in the medium, a polymer yield of more than $85 \%$ could be reached. Tapioca starch gave the highest conversion rate, and the polymer yield from tapioca was also generally the highest. (Fig. 1).

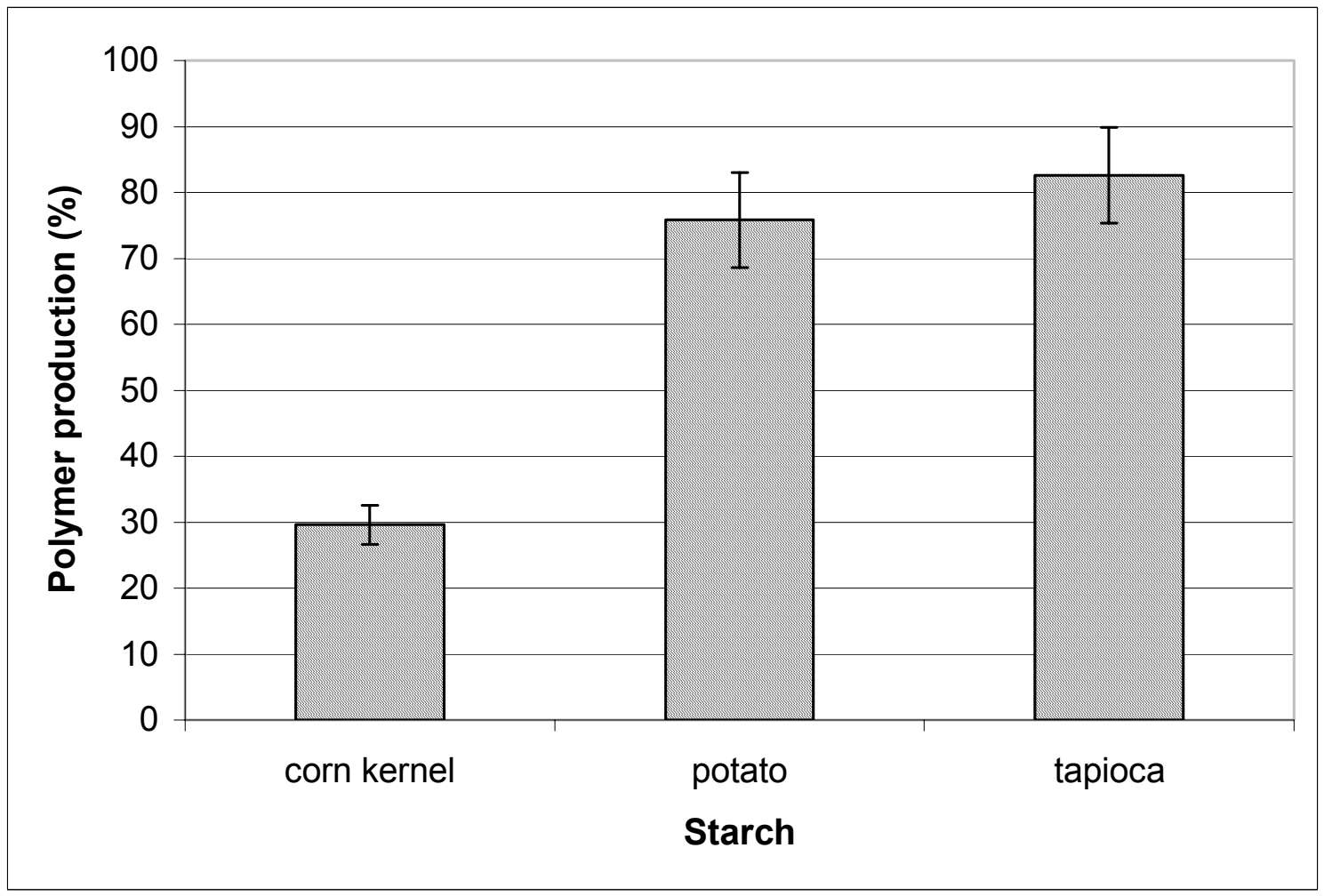

Fig. 1. Biopolymer production with different starches as raw materials after 4 days (values shown as mean $+/-\mathrm{SD}, \mathrm{N}=3$ )

\section{Characterization of the Biopolymer}

\section{Molecular weight and its distribution}

Since the primary purpose of starch modification is to produce packaging material, the molecular weight and MW distribution of the biopolymer should be important indicators of its mechanical properties. Studies on the effect of molecular weight on biopolymer film characteristics have reported that the mechanical strength of certain films increased with increasing molecular weight of the biopolymer (Nunthanid et al. 2001). This finding might be attributable to an entanglement network forming during film formation from higher molecular weight material.

In this study, the molecular weight during the modification process, from day 1 to day 4, was analyzed using the GFC column, with the results shown in Fig. 2. The "not identified" portion in the figure is the difference between the total weight of the biopolymer injected into the column and the calculated result from the GFC standard curve. The disagreement is due to the difficulty in obtaining accurate response factors over such a wide range of molecular weights. However, the GFC assay has shown clearly that the majority of the bio-polymer has a molecular weight (abbreviated $\mathrm{Mw}$ ) 
more than 8M. It was also observed that the contribution of the high $\mathrm{Mw}$ fraction increased, from $25 \mathrm{wt} . \%$ on day 1 , to $89 \mathrm{wt}$. \% on day 3 , then dropped slightly to $78 \%$ on day 4. This profile established that the modification by the fungus increased the size of the biopolymer. Based on the observations of J. Nunthanid et al. (2001), it could be speculated that the bio-polymer has a better inherent strength than that of pure starch, although the exact mechanism of the modification still remains to be determined.

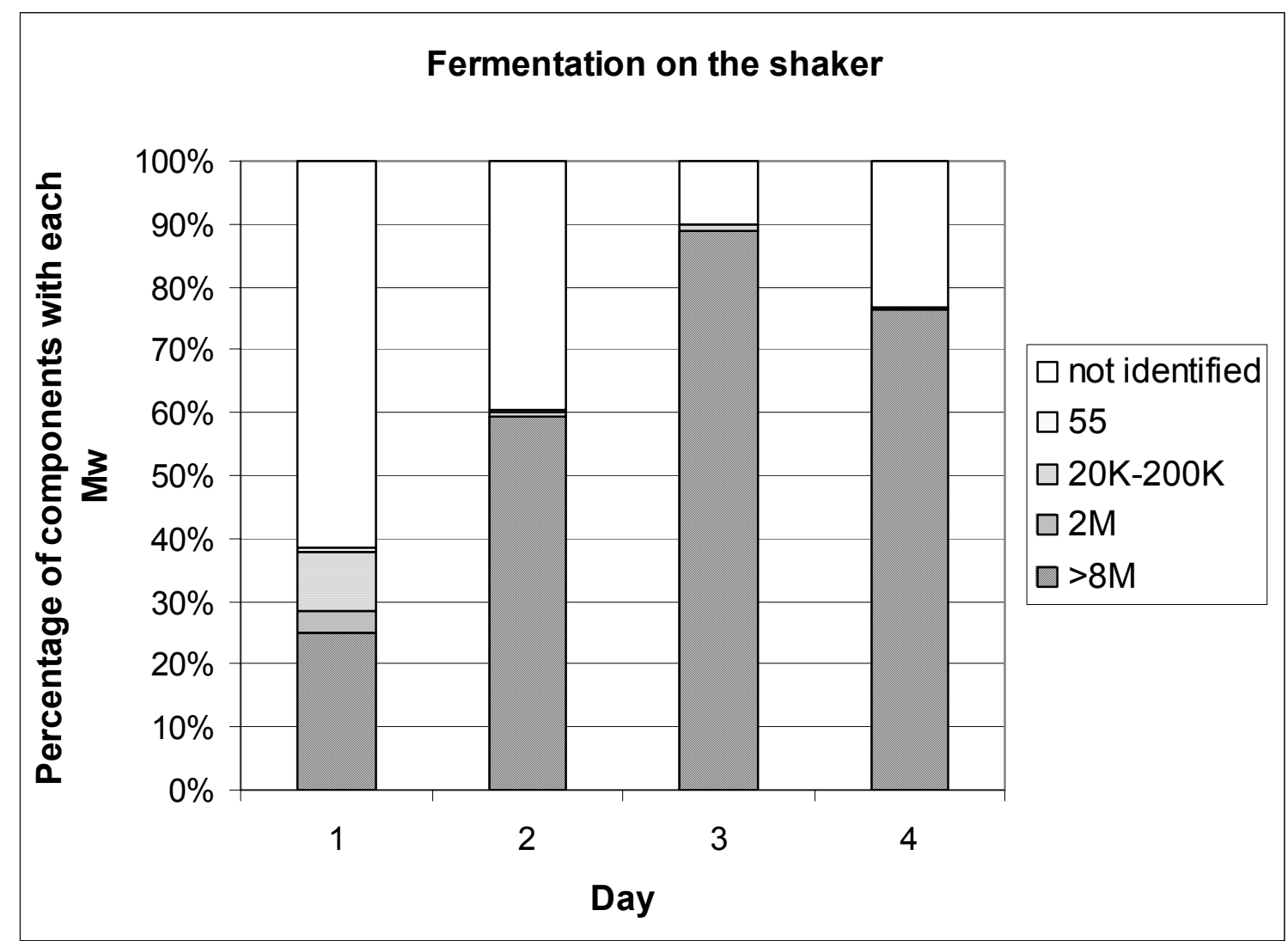

Fig. 2. Molecular weight analyses during the fermentation process with tapioca starch

The biopolymer produced by fungal fermentation was further characterized by acid digestion, followed with a Liquid Chromatography assay. Glucose was identified as the major degradation component. Very likely, there are one or more other components that contribute to the properties of the modification product.

The GFC profile of the pure starch is not measurable because of its extremely low solubility. But after modification, the biopolymer possesses greater water solubility than the original starch substrate, even though the solubility of the biopolymer varies, depending on the different starch sources.

\section{$F T-I R$}

The molecular level changes during the modification process were studied by FTIR, as shown in Fig. 3. The spectra a and $b$ represent the native potato starch and the biopolymer harvested from the fungal modification of starch, respectively. 


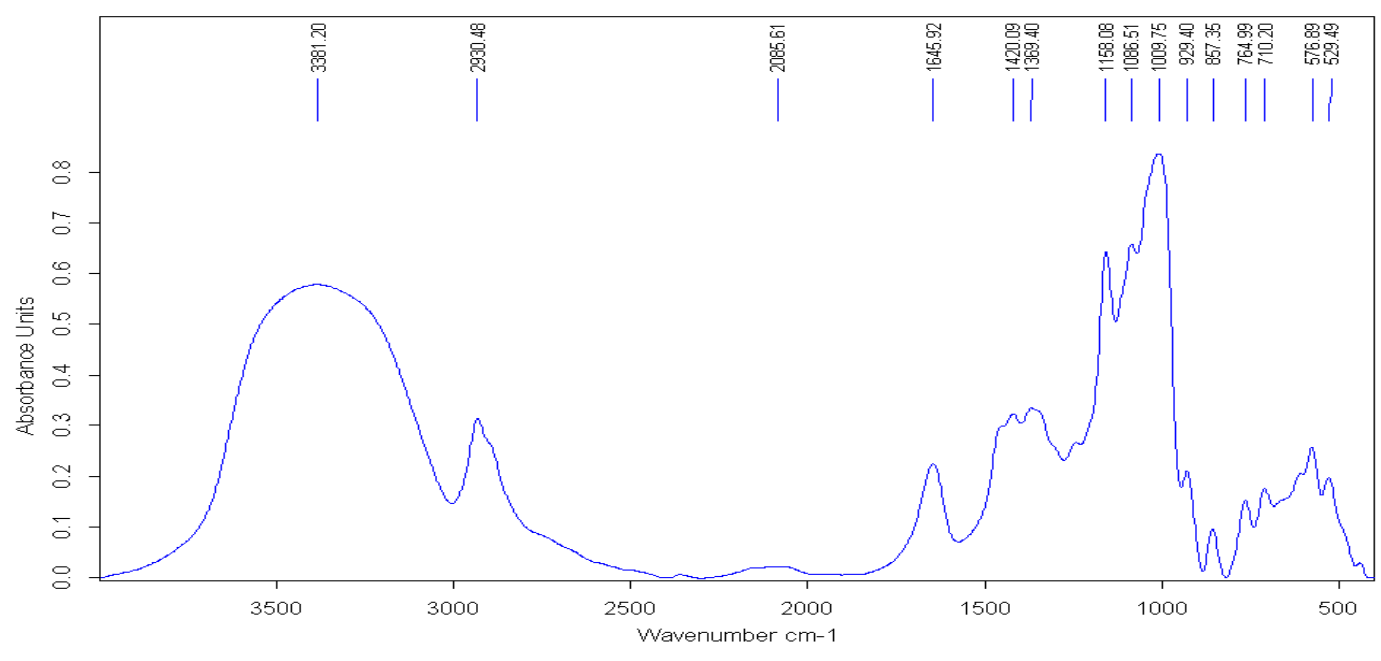

a: Native potota starch

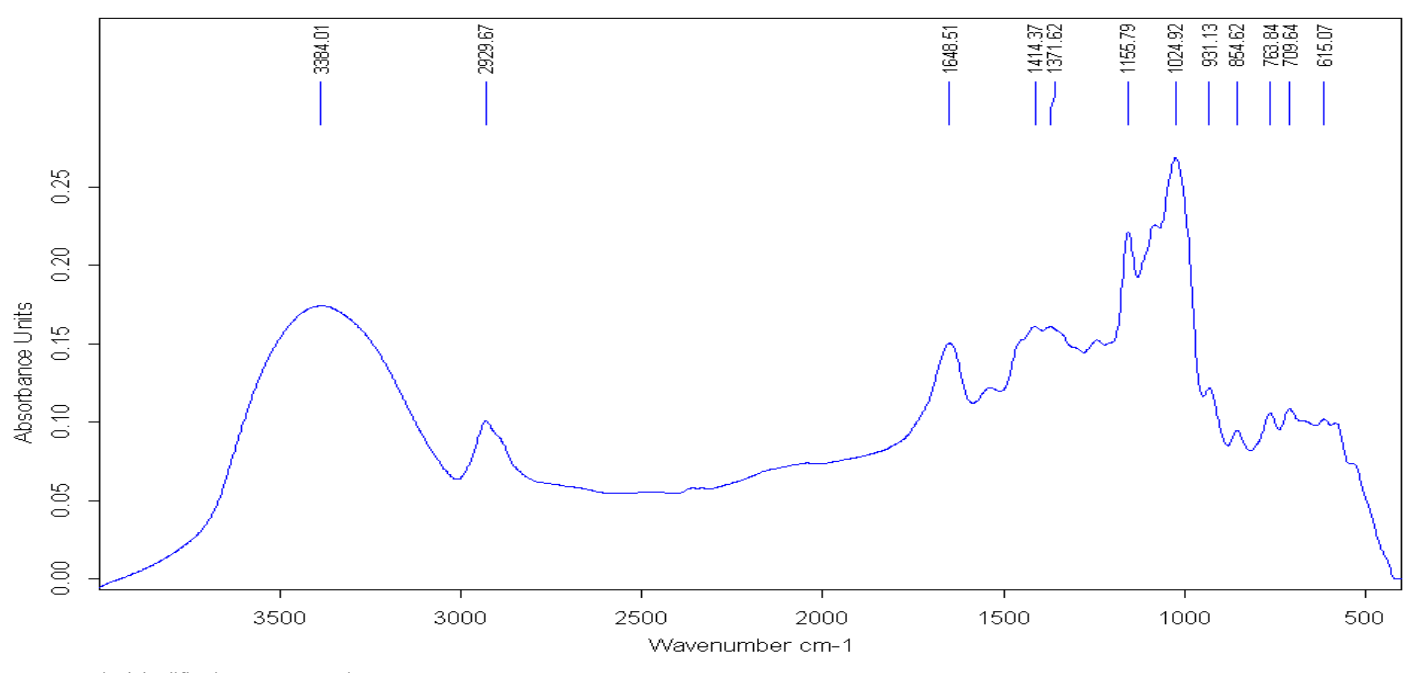

b: Modified potato starch

Figure 3. FT-IR spectra of starch and modified starch

Both spectra in the region below $800 \mathrm{~cm}^{-1}$ exhibited complex vibrational modes due to the skeletal mode vibrations of the pyranose ring in the glucose unit (Kizil et al. 2002). In the starch fingerprint region of $800 \mathrm{~cm}^{-1}$ to $1500 \mathrm{~cm}^{-1}$, spectral characteristics of the starch and modified starch were similar. However, distinctive changes could also be found. Peaks at around $1080 \mathrm{~cm}^{-1}$ to $1158 \mathrm{~cm}^{-1}$ were characteristic of a C-O-H bond. Compared the peaks in native starch with wavenumber 1158 and $1081 \mathrm{~cm}^{-1}$, they were consistently observed shift to $1156 \mathrm{~cm}^{-1}$ and $1080 \mathrm{~cm}^{-1}$ after the modification. Also, the peaks between 990 and $1030 \mathrm{~cm}^{-1}$, which represent the anhydroglucose ring O-C stretch (Fanga et al. 2002), were different after fungal modification. This phenomenon may be 
related to the stability and intensity of hydrogen bonds. Pawlak et al. (2003) showed that the stability and strength of hydrogen bonds were indicative of correlative peak shifts and the peak style changes. Moreover, significant ratio changes were observed in the infrared absorption band around $1080 \mathrm{~cm}^{-1}, 930 \mathrm{~cm}^{-1}$, and $861 \mathrm{~cm}^{-1}$, consistent with changes in the glycosidic linkages in starches (Kizil et al. 2002). A characteristic peak occurred at 1644 $\mathrm{cm}^{-1}$ in native starch, which is presumably a feature of tightly bound water (Fanga et al. 2004). However, in the biopolymer, the peak shifted to $1652 \mathrm{~cm}^{-1}$ and also increased significantly in intensity. Irudayaraj et al. (2002) showed that the absorbances at around 3389 and $2930 \mathrm{~cm}^{-1}$ can be assigned to $\mathrm{O}-\mathrm{H}$ and $\mathrm{C}-\mathrm{H}$ bond stretching, respectively. The intensity and shape of the $2930 \mathrm{~cm}^{-1}$ peak in modified starch was substantially different than that of native starch. All the above observations support the conclusion that fungal fermentation has changed the structure and bonding of the starch substrates.

\section{Mechanical Properties of the Bio-polymer Films}

Both pure starch and the microbially modified biopolymer were cast into films and subjected to tensile testing. The experimental results confirmed the above prediction, i.e., that the modified starch has better strength properties, and is well suited for use as a packaging material (Fig. 4 and Table 1). The difference in the number of samples is mainly due to the availability of the sample material-some films, especially rice and amylopectin film cracked after drying, and thus, very few complete specimens could be cut from the films. The problem with film cracking was one of the reasons why films from these materials were not selected for further study. Also, if the films did not fail at the end of the test, no data could be recorded for "elongation at break". This was observed twice for potato starch, 4 times for tapioca starch, twice for rice polymer, and once for amylopectin polymer.

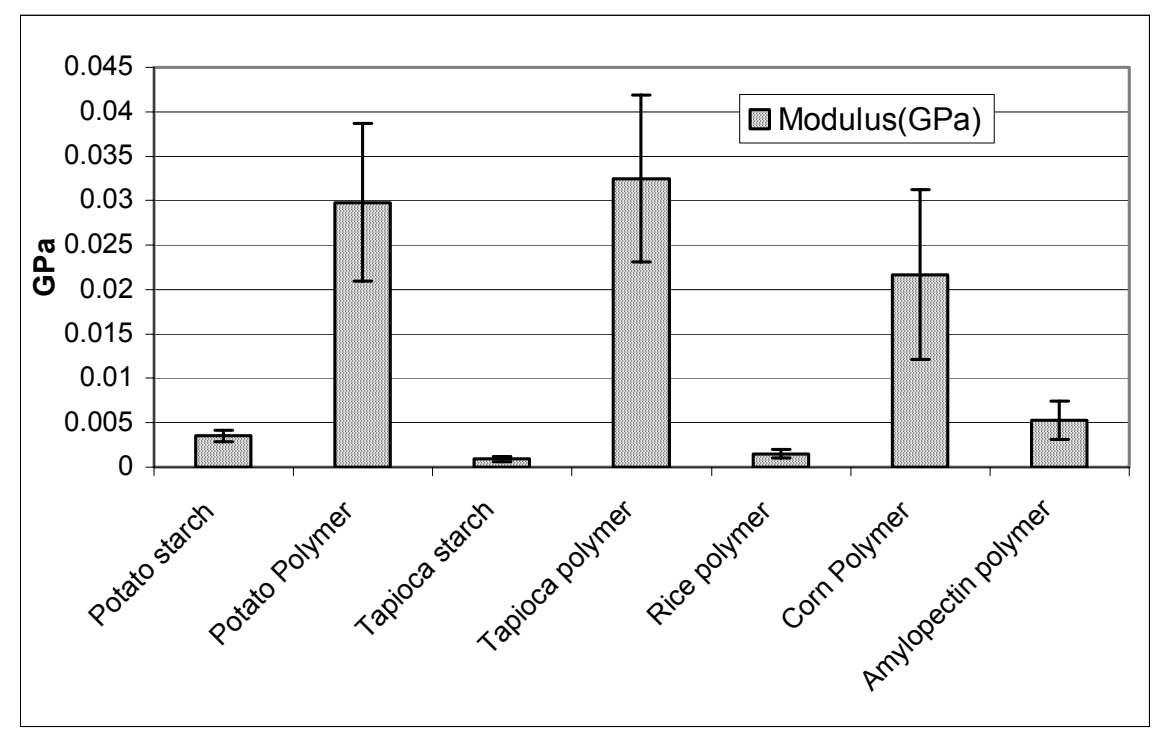

Fig. 4. Tensile modulus of the starch and the modified biopolymer films (values shown as mean $+/-\mathrm{SD}, \mathrm{N}=5,4,6,6,10,4,3$, respectively) 


\section{Table 1. Tensile Tests of Various Starches and the Fungal Modified} Biopolymer Films

\begin{tabular}{|c|c|c|c|c|c|c|}
\hline & \multicolumn{2}{|l|}{ Material } & \multirow{2}{*}{$\begin{array}{r}\text { Mean } \\
1.60\end{array}$} & \multicolumn{2}{|c|}{$\begin{array}{l}95 \% \text { confidence limits of } \\
\text { the mean }\end{array}$} & \multirow{2}{*}{\begin{tabular}{l}
$\begin{array}{l}\mathbf{N} \\
\text { (number } \\
\text { of } \\
\text { measure } \\
\text { ments) }\end{array}$ \\
\multicolumn{1}{c}{5}
\end{tabular}} \\
\hline \multirow{7}{*}{$\begin{array}{l}\text { Peak Stress } \\
\text { (MPa) }\end{array}$} & \multirow{2}{*}{ Potato } & Starch & & 1.18 & 2.01 & \\
\hline & & Polymer & 3.58 & 3.22 & 3.92 & 7 \\
\hline & \multirow{2}{*}{ Tapioca } & Starch & 0.37 & -0.01 & 0.75 & 6 \\
\hline & & Polymer & 3.60 & 3.30 & 3.89 & 10 \\
\hline & Rice & Polymer & 0.43 & -0.04 & 0.89 & 4 \\
\hline & Corn & Polymer & 2.52 & 2.14 & 2.90 & 6 \\
\hline & Amylopectin & Polymer & 0.97 & 0.44 & 1.51 & 3 \\
\hline \multirow{7}{*}{$\begin{array}{l}\text { Elongation } \\
\text { at break } \\
(\mathrm{mm})\end{array}$} & \multirow{2}{*}{ Potato } & Starch & 40.78 & 37.05 & 44.50 & 3 \\
\hline & & Polymer & 10.78 & 8.34 & 12.31 & 7 \\
\hline & \multirow{2}{*}{ Tapioca } & Starch & 48.33 & 43.76 & 52.89 & 2 \\
\hline & & Polymer & 10.77 & 8.73 & 12.81 & 10 \\
\hline & Rice & Polymer & 34.79 & 30.22 & 39.35 & 2 \\
\hline & Corn & Polymer & 13.36 & 10.73 & 16.00 & 6 \\
\hline & Amylopectin & Polymer & 21.72 & 17.16 & 26.28 & 2 \\
\hline
\end{tabular}

Statistical analysis was conducted using SAS software to generate $95 \%$ confidence limits for the mean values of peak stress and elongation at break for different materials before/after the modification. The results showed that the polymers produced by fungal modification of tapioca and potato starch possessed superior mechanical strength compared to rice and amylopectin. When compared to their native starch counterparts, a great improvement in the peak stress was obtained at a $99.9 \%$ confidence level for both potato and tapioca, with a reduction in elongation at the breakpoint. For instance, fungal modification of the tapioca starch increased its peak strength from 0.37 $\mathrm{MPa}$ to $3.50 \mathrm{MPa}$, demonstrating a huge impact of the microbial treatment. But at the same time, the elongation at break decreased from $48 \mathrm{~mm}$ to $12 \mathrm{~mm}$. Therefore, after fungal modification, the polymer increased in strength at the expense of ductility. Aging of the films was also an important factor influencing polymer properties; this accounts for some of the relatively large error bars in the results, which simply aggregate data from films of similar composition, without accounting for their age.

After modification, biopolymers derived from potato and tapioca starches exhibited a much lower water absorption, which indicates higher moisture resistance, a 
favorable property for packaging material applications. After soaking film samples in water, all the unmodified starch films broke into pieces within 30 minutes, and continued to absorb water. However, all films made from the modified biopolymer remained intact, even after 24 hours. Furthermore, their water uptake capacities reached a maximum in an hour, and then exhibited a plateau thereafter. The results from the film water absorption tests are shown in Fig. 5.

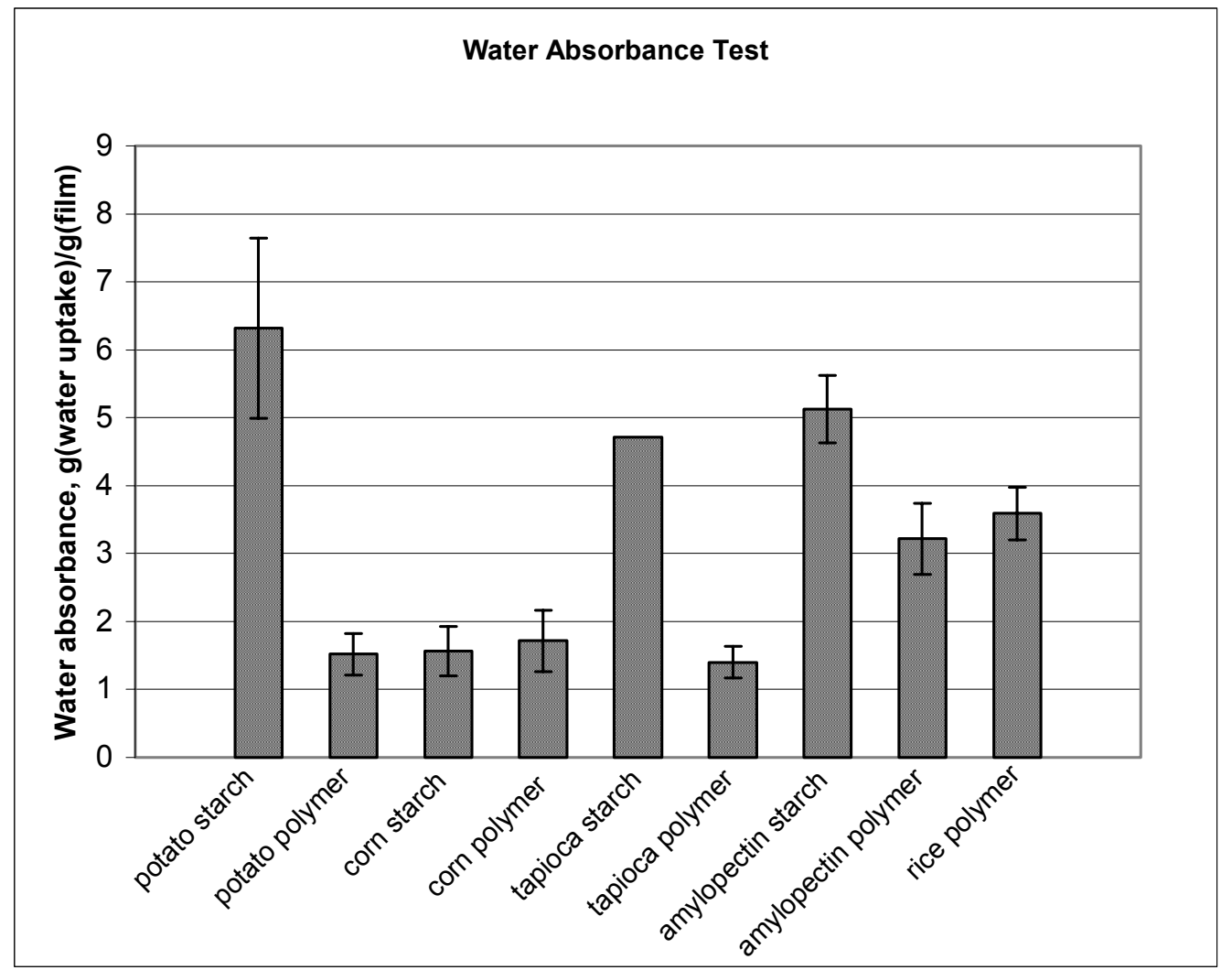

Fig. 5. Water absorbance tests for the starch and modified biopolymer films (values shown as mean $+/-\mathrm{SD}, \mathrm{N}=1 \sim 3$ )

Tensile tests were also conducted with the potato starch from the carboy fermentation, shown in Table 2 and Fig. 6. Statistical analysis of the results showed that the biopolymer quality from the third and fourth day was superior to that obtained from the earlier days of large scale fermentation. On the third day, the properties of the films were very close to those of the potato biopolymer produced in a $2 \mathrm{~L}$ lab-scale process, suggesting that the process can be readily scaled up, and supporting the concept of future mass production. The modified biopolymer has greater water solubility than the original starch, but the solubility varies, depending on the different starch sources. 
Table 2. Tensile Tests with the Time Course Fermentation of Potato Starch

\begin{tabular}{|l|c|c|c|c|c}
\hline & Day & Mean & \multicolumn{2}{|c|}{ 95\% confidence limits of the mean } & $\begin{array}{c}\text { N } \\
\text { (number of } \\
\text { measurements) }\end{array}$ \\
\hline \multirow{4}{*}{$\begin{array}{l}\text { Peak Stress } \\
\text { (MPa) }\end{array}$} & 1 & 0.58 & 0.38 & 0.77 & 5 \\
\cline { 2 - 6 } & 3 & 1.30 & 1.10 & 1.49 & 5 \\
\cline { 2 - 6 } & 4 & 2.24 & 2.04 & 2.55 & 5 \\
\hline \multirow{3}{*}{$\begin{array}{l}\text { Elongation } \\
\text { at break } \\
(\text { mm) }\end{array}$} & 2 & 8.75 & 7.74 & 2.43 & 5 \\
\cline { 2 - 6 } & 3 & 6.95 & 8.41 & 9.75 & 2 \\
\cline { 2 - 6 } & 4 & 6.29 & 6.06 & 11.23 & 5 \\
\hline
\end{tabular}

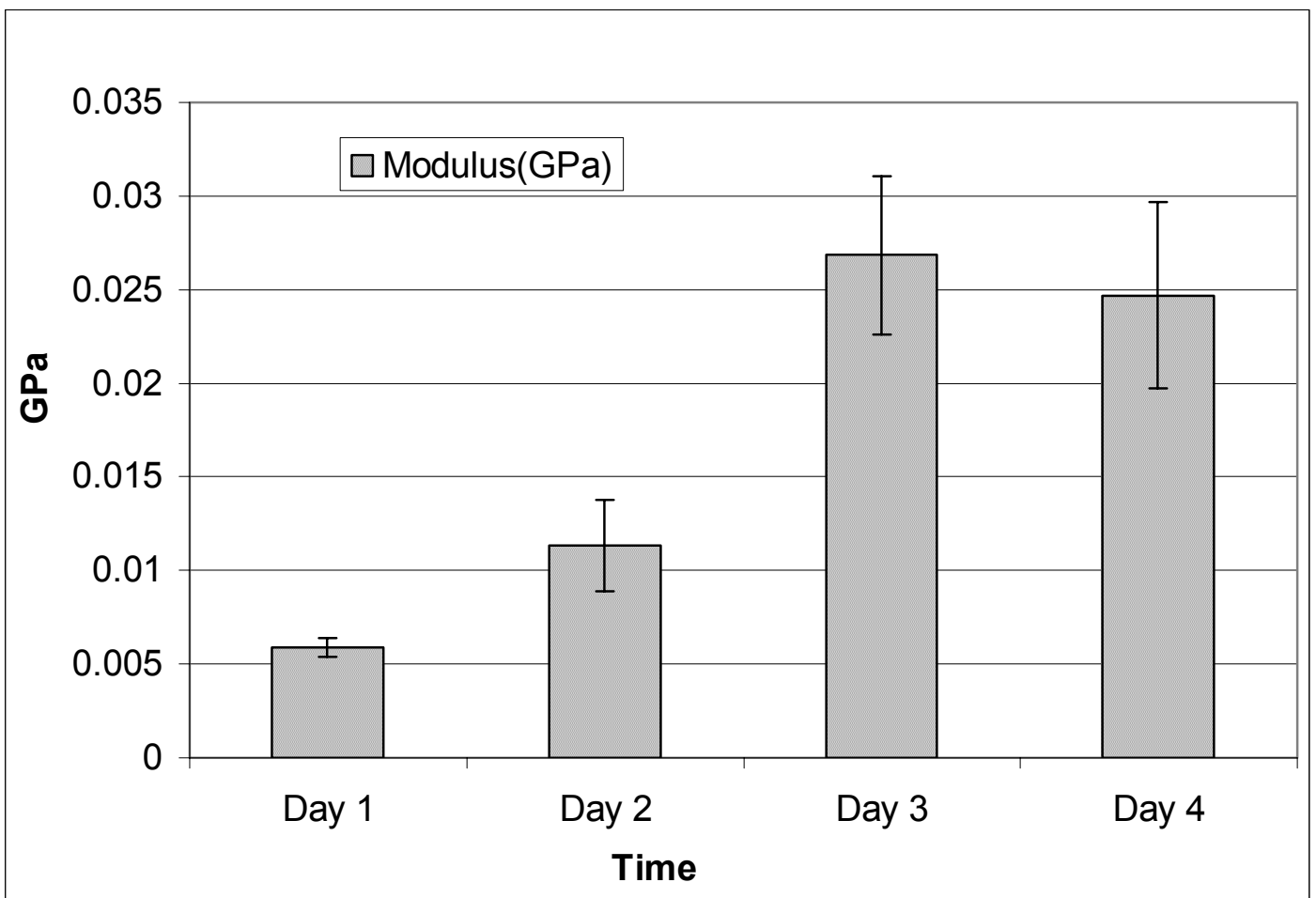

Fig. 6. Tensile modulus tests with the time course fermentation of potato starch (values shown as mean $+/-\mathrm{SD}, \mathrm{N}=5$ ) 
The overall results proved that over 72 hours, the fungus $O$. spp. is able to modify starch into a biopolymer with a higher molecular weight and greater mechanical strength. Changes in chemical bonds were also observed through FT-IR analysis. Observations of stronger hydrogen bonding indicated the enhancement of inter-molecular strength.

\section{CONCLUSION}

The overall results proved that $O$. spp. was able to successfully modify starch into a biopolymer with improved mechanical properties in about 72 hours. Results from GFC demonstrated a substantial increase in MW during the modification process. The increasing molecular weight also contributed to the improved mechanical properties of the starch films. Changes in the starch structure were studied through FT-IR. The pyranose ring was maintained after the modification, but the hydrogen bonds between molecules intensified. Peak shifts and ratio changes suggested the fixation of new chemical functional groups or new linkages between starch molecules. Based on previous (Jeng et al. In press) and current studies of the fungus, two possible pathways of the modification could therefore be proposed. One pathway involves the fungus producing a polymer that can bond starch molecules together and form new crosslinked structures. The second possible pathway involves the fungus attaching one or more functional groups that help to strengthen the starch polymer.

Work is under way to increase the understanding of the fungal modification process-a key step prior to commercial development. As more is learned about the structure-function relationships of the biopolymer, opportunities for commercial application will surely increase.

\section{ACKNOWLEDGEMENTS}

Funding from BIOCAP Canada Foundation and NSERC is deeply appreciated. We thank M. Dumas for the kind donation of the Ophiostoma spp. fungus.

\section{REFERENCES CITED}

Fanga, J. M., Fowler, P. A., Tomkinson, J., and Hill, C. A. S. (2002). "The preparation and characterisation of a series of chemically modified potato starches," Carbohydrate Polymers, 47(3), 245-252.

Fanga, J.M., Fowlera, P.A., Sayersb, C. and Williams P.A. (2004), "The chemical modification of a range of starches under aqueous reaction conditions," Carbohydrate Polymers, 55 (2004), 283-289.

Fama, L, A., Rojas, M., Goyanes, A. and Gerschenson, L. (2005), "Mechanical properties of tapioca-starch edible films containing sorbates," $L W T, 38,631-639$. 
Irudayaraj, J., and Yang, H. (2002), "Depth profiling of a heterogeneous food-packing model using step-scan Fourier transform infrared photoacoustic spectroscopy," Journal of Food Engineering, 55, 25-33.

Jeng, R., Sain, M., Hubbes, M., Saville, B. and Huang, C. (In press), "Starch-like exopolysaccharide produced by the filamentous fungi Ophiostoma ulmi and O. novoulmi," Forest Pathology, in press.

Jobling, S. (2004), "Improving starch for food and industrial applications," Plant Biology, $7(2004), 210-218$.

Kizil, J. and Seetharaman, K. (2002), "Characterization of Irradiated Starches by Using FT-Raman and FTIR Spectroscopy," Journal of Agriculture and food chemistry, 50, 3912-3918.

Laohakunjit, N., and Noomhorm, A. (2003), "Effect of plasticizers on mechanical and barrier properties of rice starch film," Starch, 56 (2004), 348-356.

Lawton, J.W. (1996), "Effect of starch type on the properties of starch containing films," Carbohydrate Polymers, 29 (1996), 203-208.

Lorcks, J. (1997), "Properties and applications of compostable starch-based plastic material," Polymer Degradation and Stability, 59 (1998), 245-249.

Mali, S., Victoria, M., Grossmann, E., M. A. Garcia, M. A., Martino ,M. N. and Zaritzky, N. E (2004) "Mechanical and thermal properties of yam starch films," Food Hydrocolloids, 19 (2005), 157-164.

Nunthanid, J., Puttipipatkhachorn, S., Yamamoto, K. and Peck G. E. (2001). "Physical properties and molecular behavior of chitosan films," Drug Development and Industrial Pharmacy Industry Pharmacy, 27(2001), 143-157.

Pawlak, A., and Mucha, M. (2003). "Thermogravimetric and FTIR studies of chitosan blends," Thermochimica Acta, 396, 153-166.

Poutanen, K. and Forssell, P. (1996). "Modification of starch properties with plasticizers," TRIP 4-4 (1996), 128-132.

Selbmann, L., Stingle F., and Petruccioli, M. (2003). "Exopolysaccharide production by filamentous fungi: the example of Botrysphaeria rhodina," Antonie Van Leeuwenhoek, 84 (2003), 135-145.

Soest, J. V., Vliegenthart, G. J. and J. F. G. (1997). "Crystallinity in starch plastics: consequences for material properties," Trends in Biotechnology, 15(6), 208-213.

Weber, C. J., Haugaard, V., Festersen, R., and Bertelsen, G. (2001). "Production and applications of biobased packaging materials for the food industry," Food Additives and Contaminants, 19, Supplement, 172-177.

Article submitted: Sept. 21, 2006; First round of reviews completed: Oct. 15, 2006; Revision accepted: Nov. 15, 2006; Article published: Nov. 24, 2006. 